\begin{tabular}{|c|l|}
\hline Title & Third stable branch and tristability of nuclear spin polarizations in a single quantum dot system \\
\hline Author(s) & Y amamoto, S.; Kaji, R.; Sasakura, H.; A dachi, S. \\
\hline Citation & $\begin{array}{l}\text { Physical Review B, 101(24), 245424 } \\
\text { https://doi.org/10.1103/PhysRevB.101.245424 }\end{array}$ \\
\hline Issue Date & 2020-06-17 \\
\hline Doc URL & http://hdl.handle.net/2115//8974 \\
\hline Rights & Copyright (2020) by The A merican Physical Society. \\
\hline Type & article \\
\hline File Information & PhysRevB.101.245424.pdf \\
\hline
\end{tabular}

Instructions for use 


\title{
Third stable branch and tristability of nuclear spin polarizations in a single quantum dot system
}

\author{
S. Yamamoto, ${ }^{*}$ R. Kaji, H. Sasakura, and S. Adachi $\odot^{\dagger}$ \\ Division of Applied Physics, Hokkaido University, N13 W8, Kitaku, Sapporo 060-8628, Japan
}

(Received 23 March 2020; revised manuscript received 25 May 2020; accepted 3 June 2020; published 17 June 2020)

\begin{abstract}
Semiconductor quantum dots provide a spin-coupled system of an electron and nuclei via enhanced hyperfine interaction. We showed that the nuclear spin polarization in single quantum dots can have three stable branches under a longitudinal magnetic field. The states were accompanied by hysteresis loops around the boundaries of each branch with a change in the excitation condition. To explain these findings, we incorporated the electron spin relaxation caused by the nuclear spin fluctuation into the previously studied dynamic nuclear spin polarization mechanism. The model reproduces the features of nuclear spin polarization and the associated strong reduction in the observed electron spin polarization, and can refer to the tristability of nuclear spin polarization.
\end{abstract}

DOI: 10.1103/PhysRevB.101.245424

\section{INTRODUCTION}

In semiconductor quantum dots (QDs), the strong localization of electron wave function enhances hyperfine interaction (HFI). Simultaneous spin-flip via HFI transfers the spin angular momentum from an electron to the lattice nuclear spin ensemble, and thus, nuclear spin polarization (NSP) can be established by spin-polarized electron injection [1-3]. The unparalleled spin coherence of nuclei has always been attractive for application to quantum memory [4] and recent demonstrations and predictions using spin waves suggest the possibility of quantum read-write processes with over $90 \%$ accuracy $[5,6]$. On the other hand, the nuclear spin fluctuation causes a serious electron spin decoherence, which is an undesired aspect of spin coupling via HFI [7]. Recent studies have reported that this electron spin decoherence can be eliminated by using the spin refocusing technique $[8,9]$.

One of the most interesting properties of NSP is bistability; the NSP transits abruptly between two stable coexisting branches due to the negative and positive feedback of the spin transfer rate [10-14]. This phenomenon occurs when electron spin splitting, which limits the spin transfer rate, is reduced by the compensation of the external magnetic field by an effective field originating from NSP (i.e., nuclear field).

In this paper, we show that NSP in individual QDs potentially has three stable branches. This intriguing behavior was demonstrated by steady-state photoluminescence (PL) measurements under nonresonant excitation and it can be explained by a phenomenological rate equation, including the effects of nuclear spin fluctuation. In addition, the proposed model predicts that a QD spin system exhibits a tristable response under some proper conditions. Our findings remind us of the degree of complication of spin coupling via HFI in a QD, which sometimes causes unintuitive behaviors, such as the bidirectional NSP formation $[15,16]$ and the anomalous

\footnotetext{
*yama-st@eis.hokudai.ac.jp

†adachi-s@eng.hokudai.ac.jp
}

Hanle effect $[17,18]$. The revealed fact related to nuclear spin fluctuation contributes to a deeper understanding of electron spin decoherence and a hybrid quantum system composed of an electron and nuclei.

\section{SAMPLE AND APPARATUS}

We used single $\mathrm{In}_{0.75} \mathrm{Al}_{0.25} \mathrm{As} / \mathrm{Al}_{0.3} \mathrm{Ga}_{0.7} \mathrm{As}$ selfassembled (SA) QDs grown by molecular-beam epitaxy on $(100) \mathrm{GaAs}$ substrate $[2,13,18,19]$. The atomic force microscopy of a reference uncapped QD layer and cross-section transmission electron microscope observation revealed a lens-like shape of QD with the typical diameter of $20 \mathrm{~nm}$ and the height of $4 \mathrm{~nm}$. The fabrication of small mesa structures enables us to access single QDs by micro-PL measurements.

Figure 1(a) shows schematics of the experimental apparatus. A cw Ti:Sapphire laser was employed to inject the spin-polarized electron and hole into the wetting layer of the QDs $(\sim 1.6986 \mathrm{eV})$. The polarization of the excitation beam was controlled with a combination of a linear polarizer (LP), a half-wave plate (HWP), and a quarter-wave plate (QWP) [20].

The excitation beam was focused on the QD sample along the growth direction $(z)$ through an objective lens (OL, Mitsutoyo M Plan Apo NIR $\times 20$ ). Our sample was kept at $6 \mathrm{~K}$ in an optical cryostat. A superconducting magnet (Oxford Instruments, MicrostatBT) introduced a static longitudinal magnetic field $B_{z}$ up to $5 \mathrm{~T}$. The PL from the individual QDs collected by the same OL was dispersed by a triple grating spectrometer (Horiba JY, T64000) and detected by a liquid-nitrogen cooled charge-coupled device (CCD, Princeton Instruments, Spec10:100BR). Although the energy resolution of our apparatus was $\sim 12 \mu \mathrm{eV}$, the peak energy could be determined with an accuracy of $\lesssim 5 \mu \mathrm{eV}$ by using the spectral fittings.

We analyzed the PL spectra of three different single QDs (QD1, QD2, and QD3). Figure 1(b) [1(c)] shows the typical PL spectra from QD1 [QD2 and QD3] which was detected in a linearly polarized basis at $B_{z}=0$. In this paper, we focus on a positively charged exciton $X^{+}$that consists of one electron and two holes in a spin singlet. The degree 


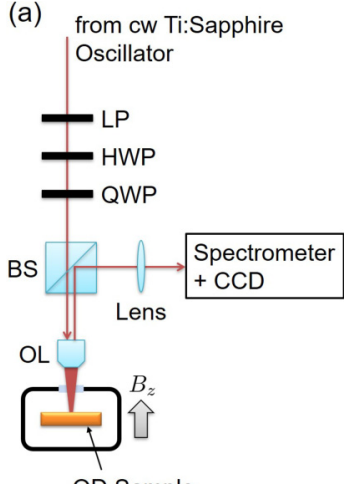

QD Sample
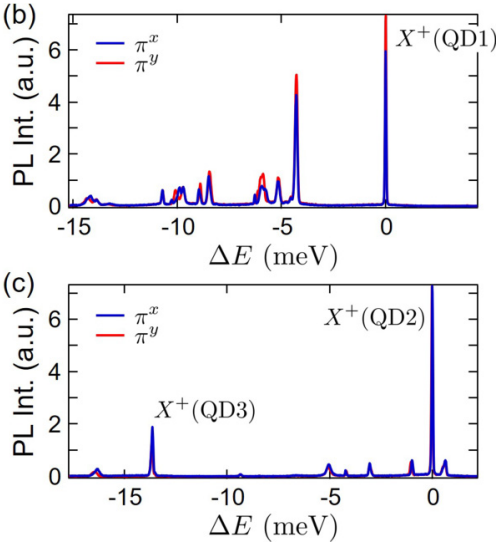

FIG. 1. (a) Schematics of the experimental apparatus. BS: Beam splitter. (b), (c) PL spectra from single InAlAs SA QDs. The origin of the horizontal axis $\Delta E$ is 1.6264 (1.6405) $\mathrm{eV}$ in (b) [(c)] which is the peak energy of $X^{+}$PL of QD1 (QD2). In this paper we focus on the $X^{+}$spectra that does not show the fine structure splitting.

of circular polarization of $X^{+}$PL depends only on the $z$ component of electron spin polarization $\left\langle S_{z}\right\rangle$ and is written as $\rho_{\mathrm{c}}=2\left\langle S_{z}\right\rangle$, where $\rho_{\mathrm{c}}=\left(I^{-}-I^{+}\right) /\left(I^{-}+I^{+}\right)$and $I^{-(+)}$denotes the intensity of $\sigma^{-(+)}$polarized PL. The established NSP $\left\langle I_{z}\right\rangle$ along the $z$ axis was monitored through the Overhauser shift $\Delta_{\mathrm{OS}}$ defined as $2 \tilde{A}\left\langle I_{z}\right\rangle$, where $\tilde{A}$ is a hyperfine constant $\sim 50 \mu \mathrm{eV}[21,22]$.

Note that almost all PL peaks on the lower energy side of $X^{+}$in Fig. 1(b) did not originate from QD1. Further, in all studied QDs, the intensity of $X^{+}$PL was quite larger than that of the other exciton complexes such as neutral exciton. Thus we assume that the nuclear spin pumping is dominantly accomplished via $X^{+}$, and only electron in the $X^{+}$state will be considered in the following discussions.

\section{RESULTS AND DISCUSSION}

\section{A. Observation of three stable branches}

The excitation polarization dependence of the $X^{+}$PL spectra obtained from QD1 at $B_{z}=+3.0 \mathrm{~T}$ is shown in the top panel of Fig. 2(a) as a color-scale plot. Because the $g$ factor $g_{\mathrm{e}}$ of the conduction electron is positive in our InAlAs QDs [23], the compensation of $B_{z}$ by a nuclear field $B_{\mathrm{n}, z}$ occurred under $\sigma^{+}$excitation, where the spin-down electron is photo-injected selectively. In case of such a condition, the PL energies and intensities indicated abrupt changes. To closely examine this point, both the $\rho_{\mathrm{c}}$ and $\Delta_{\mathrm{OS}}$ obtained from the spectra are plotted in the middle and bottom panels, respectively. It should be noted that there are two-stage abrupt jumps, which suggest the presence of three stable branches labeled Lo., Mid., and Hi. in the figure.

We also found three such stable branches of NSP by changing the excitation power $P_{\mathrm{exc}}$. Figure 2(b) shows the $\rho_{\mathrm{c}}$ and $\Delta_{\mathrm{OS}}$ obtained from QD2 at $B_{z}=+5.0 \mathrm{~T}$ under $\sigma^{+}$excitation. In the figure, the filled and open circles (squares) indicate the observed $\rho_{\mathrm{c}}\left(\Delta_{\mathrm{OS}}\right)$ with increasing and decreasing $P_{\mathrm{exc}}$, respectively. With increasing $P_{\text {exc }},\left|\rho_{\mathrm{c}}\right|$ and $\left|\Delta_{\mathrm{OS}}\right|$ jumped simultaneously at $\sim 150 \mu \mathrm{W}\left(P_{\text {max }}^{\mathrm{I}}\right)$ and an additional abrupt change occurred at $\sim 350 \mu \mathrm{W}\left(P_{\max }^{\mathrm{II}}\right)$ again. The similar behavior
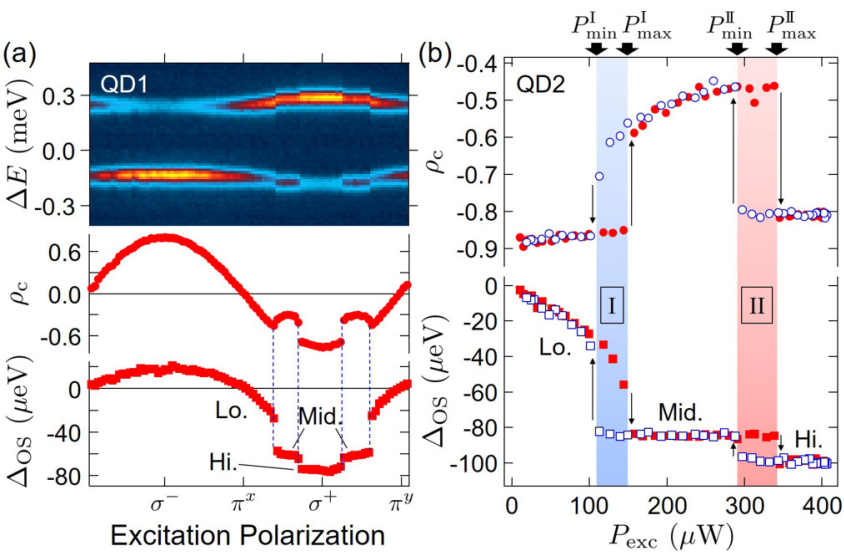

FIG. 2. (a) Excitation polarization dependence of $X^{+}$PL from QD1 at $B_{z}=+3.0 \mathrm{~T}$ and $6 \mathrm{~K}$. Top panel is a color-scale plot of PL spectra whose vertical axis $\Delta E$ indicates the PL energy from $1.6264 \mathrm{eV} . \rho_{\mathrm{c}}$ (middle) and $\Delta_{\mathrm{OS}}$ (bottom) are obtained from the data in the top panel by the spectral fitting. (b) $P_{\text {exc }}$ dependencies of $\rho_{\mathrm{c}}$ (top) and $\Delta_{\mathrm{OS}}$ (bottom) of QD2 at $B_{z}=+5.0 \mathrm{~T}$ under $\sigma^{+}$ excitation. Filled (open) symbols indicate the results with increasing (decreasing) $P_{\text {exc }}$.

with two critical points $\left(P_{\min }^{\mathrm{II}}\right.$ and $\left.P_{\min }^{\mathrm{I}}\right)$ was observed with decreasing $P_{\text {exc }}$. Notably, $P_{\min }^{\mathrm{I}(\mathrm{II})}$ was lower than $P_{\max }^{\mathrm{I}(\mathrm{II})}$. That is to say, hysteresis loops were observed around each boundary of the branches [loop I (II) for the boundary between the Lo. and Mid. (the Mid. and Hi.) branches].

Note that the Mid. branch is not always observed; its appearance depends on the QD properties and experimental conditions. For example, Fig. 3(a) shows $P_{\text {exc }}$ dependencies of the $\rho_{\mathrm{c}}$ and $\Delta_{\mathrm{OS}}$ of QD3 at $B_{z}=+5.0 \mathrm{~T}$. Although the experimental condition was the same as in the case of Fig. 2(b), the Mid. branch did not appear, and only a single hysteresis loop was observed. Further, QD2 revealed the bistability in $B_{z}=$ +3.0 T, as shown in Fig. 3(c), as well as QD3 in Fig. 3(b). Figures 3(d) and 3(e) show the $P_{\text {exc }}$ dependencies of $\Delta_{\mathrm{OS}}$ and $\rho_{\mathrm{c}}$ at various $B_{z}$ obtained from QD2. The width of loop II and the region corresponding to the Mid. branch reduced with decreasing $B_{z}$, and the Mid. branch disappeared completely at a $B_{z}$ smaller than $+4.0 \mathrm{~T}$. Although the presence of the Mid. branch could not be judged distinctively from the change in $\Delta_{\mathrm{OS}}$ at $\sim+4.0 \mathrm{~T}$, a reduction of $\left|\rho_{\mathrm{c}}\right|$ in the Mid. branch was more obvious compared with the other two branches and was the most prominent indication of the Mid. branch.

To examine the difference between the bistable and the three stable cases, we focus on the compensation point and the magnitude relation between the nuclear field $B_{\mathrm{n}, z}$ and $B_{z}$ in each branch. The compensation point, where $B_{\mathrm{n}, z}+B_{z}$ becomes zero, is explicitly reflected on the reduction of $\left|\rho_{\mathrm{c}}\right|$; hence, $\left|\left\langle S_{z}\right\rangle\right|$. This is because the electron spin relaxation, which is strongly suppressed by the Zeeman splitting, is enhanced at the compensation condition due to the degeneracy of electron Zeeman states.

In the bistable case, as shown in Figs. 3(a)-3(c), the lowest value of $\left|\rho_{\mathrm{c}}\right|$ was realized at $P_{\min }$, where $\left|\Delta_{\mathrm{OS}}\right|$ drops with decreasing $P_{\text {exc }}$. Thus, $P_{\min }$ of the Hi. branch was considered to be the compensation point. Since $B_{\mathrm{n}, z}$ usually continues to grow with increasing $P_{\text {exc }}$, as long as the system stays the 

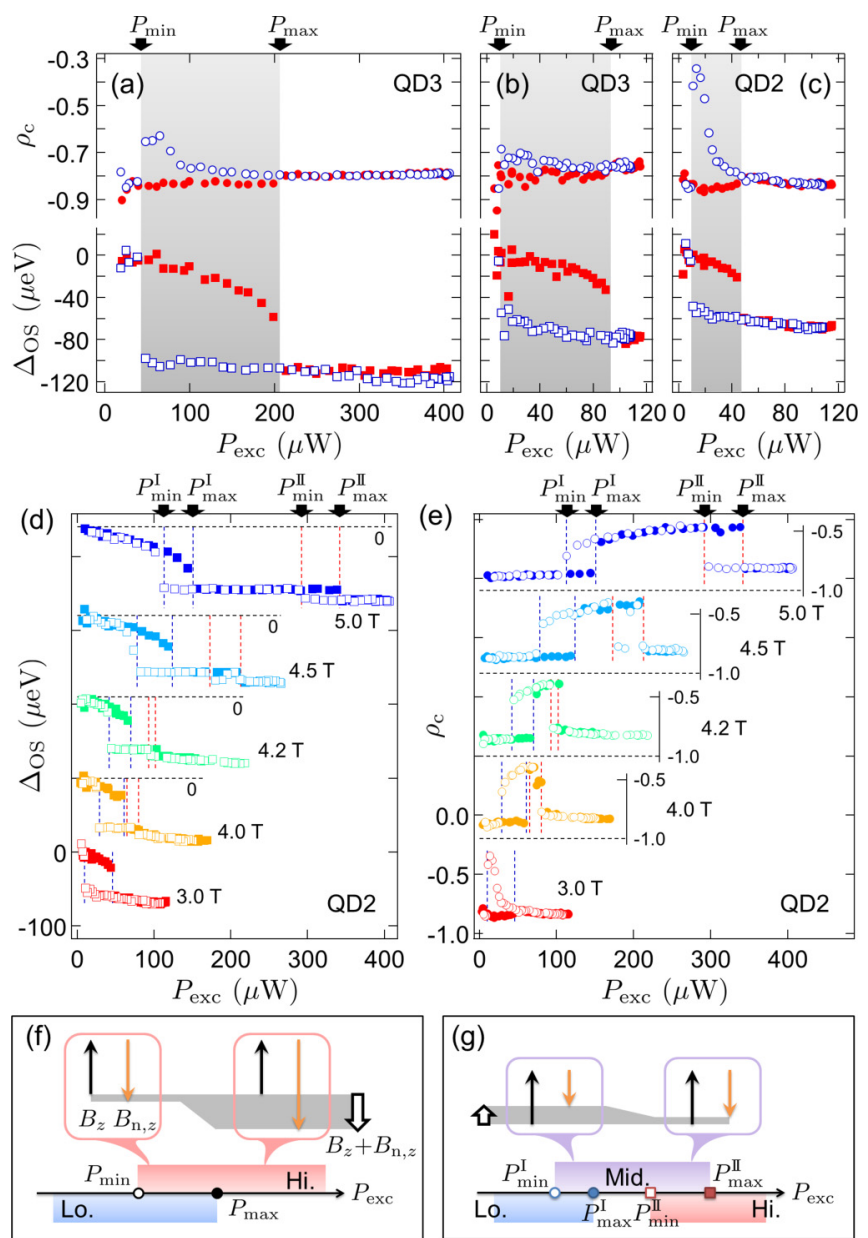

FIG. 3. (a)-(c) $P_{\text {exc }}$ dependencies of $\rho_{\mathrm{c}}$ and $\Delta_{\mathrm{OS}}$ (top and bottom panels) under $\sigma^{+}$excitation. (a) and (b) are observed in QD3 at $B_{z}=$ $+5.0 \mathrm{~T}$ and $+3.0 \mathrm{~T}$, respectively, and (c) is observed in QD2 at $B_{z}=$ $+3.0 \mathrm{~T}$. (d), (e) $P_{\text {exc }}$ dependencies of $\Delta_{\mathrm{OS}}$ and $\rho_{\mathrm{c}}$ of QD2 at various $B_{z}$. The data are shifted vertically for clarity. (f), (g) Schematics of effective fields on an electron in the Hi. branch of the bistable case (f) and in the Mid. branch of the three stable branches (g). Blue, purple, and red shades indicate the $P_{\text {exc }}$ ranges of the Lo., Mid., and Hi. branches, respectively. Thin black and orange arrows depict $B_{z}$ and $B_{\mathrm{n}, z}$, respectively. The sum, $B_{z}+B_{\mathrm{n}, z}$ is represented by the open arrows and the gray shades indicate the magnitude and sign of the arrows.

same branch, the relation $\left|B_{\mathrm{n}, z}\right|>\left|B_{z}\right|$ is held in the region $P_{\text {exc }}>P_{\text {min }}$. Accordingly, the increment of $P_{\text {exc }}$ results in the increase of the total field seen by a QD electron [shown as a gray shade in Fig. 3(f)], and the restoration of $\left\langle S_{z}\right\rangle$ in the Hi. branch with increasing $P_{\text {exc }}$ is expected. This can be observed in Figs. 3(a)-3(c).

In the case with three stable branches, as shown in Fig. 2(b), on the other hand, the lowest value of $\left|\rho_{\mathrm{c}}\right|$, and hence the compensation point, appeared at $P_{\max }^{\mathrm{II}}$. At this point, $\Delta_{\mathrm{OS}}$ changes abruptly from the Mid. to the Hi. branch with increasing $P_{\text {exc }}$. Throughout the Mid. branch, since $\left|\rho_{\mathrm{c}}\right|$ decreased with increasing $P_{\text {exc }}$, the electron Zeeman splitting was considered to keep reducing as $P_{\text {exc }}$ approached $P_{\max }^{\mathrm{II}}$. This situation is achieved if $\left|B_{\mathrm{n}, z}\right|<\left|B_{z}\right|$ in the Mid. branch, as illustrated in Fig. 3(g). Therefore, the magnitude relation between $\left|B_{\mathrm{n}, z}\right|$ and $\left|B_{z}\right|$ in the Mid. branch was opposite to that in the Hi. branch.

Regarding the observed data closely, the emergence of the Mid. branch seemed to depend on the properties of the QD system. Comparing Figs. 3(b) and 3(c), for example, although both data showed a single hysteresis loop under the same $B_{z}$, the widths were significantly different, which could be attributed to the difference in the correlation time of the HFI and/or relaxation time of the NSP. In addition, the reduction of $\left|\rho_{\mathrm{c}}\right|$ at the compensation point was more significant in QD2 compared with that in QD3. This indicates that the electron spin relaxation rate in QD2 under the condition of $B_{z}+B_{\mathrm{n}, z}=$ 0 is larger than that in QD3.

\section{B. Phenomenological model for tristability}

To describe the observed Mid. branch, we develop a phenomenological model based on the dynamic formation of NSP. As with previous works [24], we assume the equation of motion for NSP $\left\langle I_{z}\right\rangle$ as

$$
\frac{d\left\langle I_{z}\right\rangle}{d t}=\frac{1}{T_{\mathrm{NF}}}\left[Q\left(\left\langle S_{z}\right\rangle-\left\langle S_{\mathrm{eq}}\right\rangle\right)-\left\langle I_{z}\right\rangle\right]-\frac{1}{T_{\mathrm{ND}}}\left\langle I_{z}\right\rangle,
$$

where $1 / T_{\mathrm{NF}}$ is the spin transfer rate from an electron to nuclei, $Q$ is a numerical factor of $\sim 16$ for our InAlAs QDs, $\left\langle S_{\text {eq }}\right\rangle$ is the electron spin polarization in the thermal equilibrium, and $1 / T_{\mathrm{ND}}$ is the relaxation rate of NSP. We assume that $\left\langle S_{\mathrm{eq}}\right\rangle$ is negligible and hereafter treated as zero. Considering the random perturbation of HFI or the energy conservation during the electron-nuclear flip-flop process, $1 / T_{\mathrm{NF}}$ is written as

$$
\begin{aligned}
\frac{1}{T_{\mathrm{NF}}} & =\frac{2 f_{\mathrm{e}} \tau_{\mathrm{c}}(\tilde{A} / N)^{2}}{\hbar^{2}+\left[\mathrm{g}_{\mathrm{e}} \mu_{\mathrm{B}}\left(B_{z}+B_{\mathrm{n}, z}^{\mathrm{stat}}\right) \tau_{\mathrm{c}}\right]^{2}} \\
& =\gamma f_{\mathrm{e}} \mathcal{L}\left(\omega_{\mathrm{e}} ; \frac{1}{\tau_{\mathrm{c}}}\right),
\end{aligned}
$$

where $\hbar$ and $\mu_{\mathrm{B}}$ are the reduced Planck's constant and the Bohr magneton, $f_{\mathrm{e}}$ is the time fraction when an unpaired electron spin occupies the QD, $N$ is the number of nuclei, $\tau_{\mathrm{c}}$ is the correlation time of HFI, and $B_{\mathrm{n}, z}^{\mathrm{stat}}=2 \tilde{A}\left\langle I_{z}\right\rangle /\left(\mathrm{g}_{\mathrm{e}} \mu_{\mathrm{B}}\right)$ is the static part of $B_{\mathrm{n}, z}$. As shown in Eq. (2b), $1 / T_{\mathrm{NF}}$ is simplified by using the definitions $\gamma=2 \tau_{\mathrm{c}}[\tilde{A} /(\hbar N)]^{2}$ and $\omega_{\mathrm{e}}=\mathrm{g}_{\mathrm{e}} \mu_{\mathrm{B}}\left(B_{z}+B_{\mathrm{n}, z}^{\mathrm{stat}}\right) / \hbar$, and the notation $\mathcal{L}(x ; w)=1 /[1+$ $\left.(x / w)^{2}\right]$ represents the Lorentzian function of $x$ with a width $w$. Note that the experimental parameter $P_{\text {exc }}$ is considered to relate with $f_{\mathrm{e}}$.

As shown in Fig. 4(a), Eq. (2a) [also Eq. (2b)] explains that the spin transfer rate has the maximum at $\omega_{\mathrm{e}}=0$ where the electron Zeeman sublevels are degenerate due to the compensation of $B_{z}$ by $B_{\mathrm{n}, z}^{\text {stat }}$. As has been clarified by previous works [10-14,24], this peak structure brings a bistable response of NSP but cannot reproduce the three stable branches as observed if $\left\langle S_{z}\right\rangle$ has a constant value.

Thus, in addition to this, we consider the electron spin depolarization which is reflected to the nuclear spin dynamics through the NSP formation term, $\left[Q\left\langle S_{z}\right\rangle-\left\langle I_{z}\right\rangle\right] / T_{\mathrm{NF}}$, in Eq. (1). With the aid of the Bloch equation, we treat the dynamics of electron spin $\boldsymbol{S}$ as

$$
\frac{d \boldsymbol{S}}{d t}=\frac{\mathrm{g}_{\mathrm{e}} \mu_{\mathrm{B}}\left(\boldsymbol{B}_{\mathrm{ext}}+\boldsymbol{B}_{\mathrm{n}}\right)}{\hbar} \times \boldsymbol{S}-\frac{1}{\tau_{\mathrm{r}}}\left(\boldsymbol{S}-\boldsymbol{S}_{\mathrm{i}}\right)-\frac{1}{\tau_{\mathrm{s}}} \boldsymbol{S} .
$$



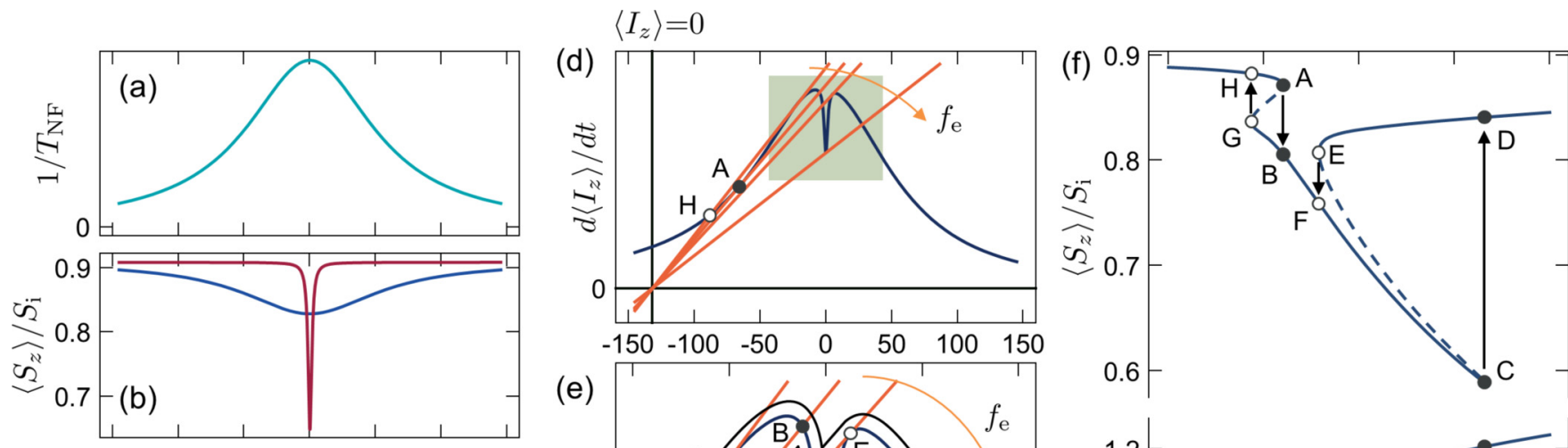

(e)

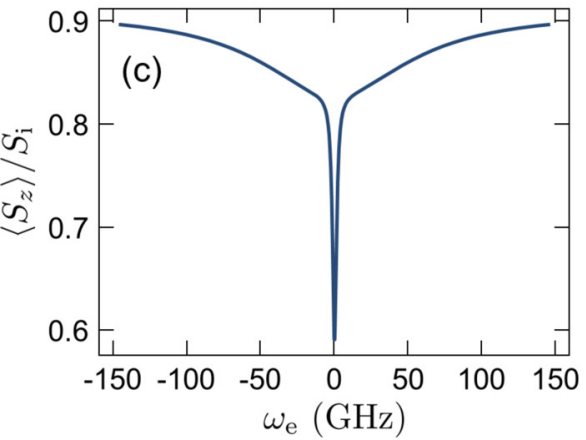

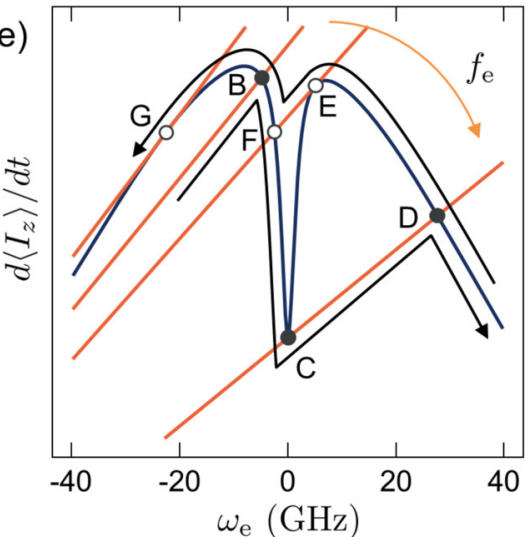

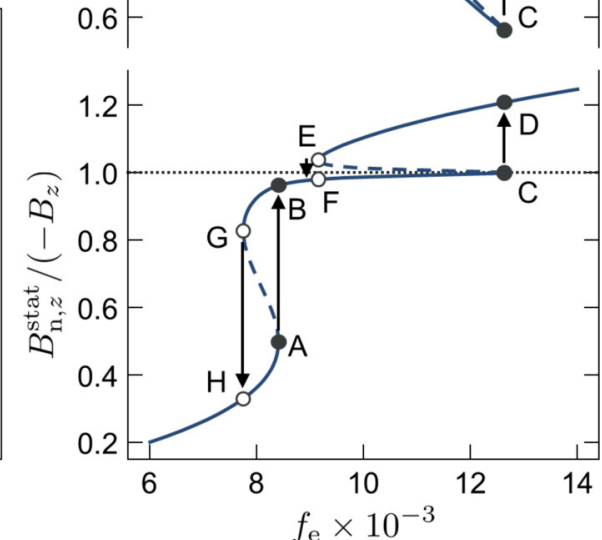

FIG. 4. (a) Spin transfer rate $1 / T_{\mathrm{NF}}$ as a function of $\omega_{\mathrm{e}} \cdot \omega_{\mathrm{e}}=0$ represents the complete compensation of the external field by the generated nuclear field. (b) Schematic display of the electron spin-flip relaxation (blue) and the nuclear spin fluctuation (red). (c) Total $\left\langle S_{z}\right\rangle$ with both the electron spin-flip relaxation and nuclear spin fluctuation. The vertical axis is normalized by $S_{\mathrm{i}}$. (d), (e) Schematics of the NSP formation (blue curve) and decay terms (orange lines) in Eq. (1). (e) is a closeup view of the square region in (d). The steady-state solutions are interpreted as the intersections of the curves, and the inclination of the decay term depends on $f_{\mathrm{e}}$. Solid (open) circles represent the intersections with increasing (decreasing) $f_{\mathrm{e}}$, and arrowed black curves indicate the state-tracking routes. (f) $f_{\mathrm{e}}$ dependencies of $\left\langle S_{z}\right\rangle$ (top) and $B_{\mathrm{n}, z}^{\text {stat }}$ (bottom). The labeled points A-H correspond to those in (d) and (e). The dashed curves indicate the unstable branches.

Here, $\boldsymbol{B}_{\text {ext }}$ is an applied magnetic field, $\boldsymbol{S}_{\mathrm{i}}$ is the spin polarization of a photo-injected electron, and $\tau_{\mathrm{r}}$ and $\tau_{\mathrm{s}}$ are the annihilation and the depolarization times of $\boldsymbol{S}$. To describe our experimental data, we put $\boldsymbol{B}_{\mathrm{ext}}=\left[0,0, B_{z}\right]$ and $\boldsymbol{S}_{\mathrm{i}}=\left[0,0, S_{\mathrm{i}}\right]$. Herein, we consider two electron spin relaxation mechanisms: spin-flip relaxation induced by HFI (hereafter called "electron spin-flip relaxation"), and spin dephasing caused by nuclear spin fluctuation.

Electron spin-flip relaxation can be regarded as the counteraction of electron-nuclei spin flip whose rate is proportional to $1 / T_{\mathrm{NF}}$. As well as the intrinsic term with a rate $1 / \tau_{\mathrm{s}}^{\text {int }}$, this process contributes the electron spin depolarization rate, and thus $1 / \tau_{\mathrm{s}}$ is written

$$
\frac{1}{\tau_{\mathrm{s}}}=\frac{1}{\tau_{\mathrm{s}}^{\text {int }}}+\frac{N Q}{f_{\mathrm{e}}} \frac{1}{T_{\mathrm{NF}}}
$$

The factor $N Q / f_{\mathrm{e}}$ comes from the fact that a single electron interacts with $N$ nuclei simultaneously $[25,26]$.

In addition, nuclear spin fluctuation causes a severe spin relaxation of localized electrons [7]. We assume that the total nuclear field $\boldsymbol{B}_{\mathrm{n}}$ can be separated as

$$
\boldsymbol{B}_{\mathrm{n}}=\boldsymbol{B}_{\mathrm{n}}^{\text {stat }}+\boldsymbol{B}_{\mathrm{f}},
$$

where $\boldsymbol{B}_{\mathrm{n}}^{\text {stat }}$ and $\boldsymbol{B}_{\mathrm{f}}$ are the static and fluctuating terms of $\boldsymbol{B}_{\mathrm{n}}$, respectively. Here, we suppose the situation that the temporal evolution of nuclear spins is much slower than that of an electron. Then, the electron spin feels a fixed (or constant) $\boldsymbol{B}_{\mathrm{n}}$ during $\tau_{\mathrm{r}}$, and thus, the influence of nuclear spin fluctuation appears only in the averaged electron spin polarization $\langle\boldsymbol{S}\rangle$. Besides, only the steady state of electron affects the nuclear spin dynamics. This is because the nuclear spins cannot follow the fast transient responses of electron. Therefore, we just consider the averaged value of the steady-state electron spin given as

$$
\left\langle S_{z}\right\rangle \approx S_{0} \frac{B_{1 / 2}^{2}+\left\langle B_{\mathrm{f}, z}^{2}\right\rangle+\left(B_{z}+B_{\mathrm{n}, z}^{\mathrm{stat}}\right)^{2}}{B_{1 / 2}^{2}+\left\langle B_{\mathrm{f}}^{2}\right\rangle+\left(B_{z}+B_{\mathrm{n}, z}^{\mathrm{stat}}\right)^{2}} .
$$

Here, $B_{1 / 2}=\hbar /\left(\mathrm{g}_{\mathrm{e}} \mu_{\mathrm{B}} T_{\mathrm{s}}\right)$ is a reduced value of the spin lifetime $T_{\mathrm{s}}=1 /\left(1 / \tau_{\mathrm{r}}+1 / \tau_{\mathrm{s}}\right)$, and $S_{0}=\left(T_{\mathrm{s}} / \tau_{\mathrm{r}}\right) S_{\mathrm{i}}$ is the upper limit of electron spin polarization. In this notation, the isotropic distribution, $\left\langle B_{\mathrm{f}}^{2}\right\rangle=3\left\langle B_{\mathrm{f}, \xi}^{2}\right\rangle(\xi=x, y, z)$, of $\boldsymbol{B}_{\mathrm{f}}$ is assumed. More details can be seen in Appendix.

The following transformation of Eq. (6) gives some prospects for multistability of NSP. The above expression can be simplified as

$$
\left\langle S_{z}\right\rangle=S_{0}\left[1-\frac{2}{3} \frac{T_{\mathrm{s}}^{2}}{T_{\Delta}^{2}+T_{\mathrm{s}}^{2}} \mathcal{L}\left(\omega_{\mathrm{e}} ; \sqrt{\frac{1}{T_{\Delta}^{2}}+\frac{1}{T_{\mathrm{s}}^{2}}}\right)\right]
$$

by using the definition

$$
S_{0}=S_{\mathrm{i}} \frac{T_{\mathrm{s}}^{\mathrm{int}}}{\tau_{\mathrm{r}}}\left[1-\frac{\gamma^{\prime} T_{\mathrm{s}}^{\text {int }}}{1+\gamma^{\prime} T_{\mathrm{s}}^{\text {int }}} \mathcal{L}\left(\omega_{\mathrm{e}} ; \frac{\sqrt{1+\gamma^{\prime} T_{\mathrm{s}}^{\text {int }}}}{\tau_{\mathrm{c}}}\right)\right] .
$$


Here, $T_{\Delta}$ represents the electron spin dephasing caused by the nuclear spin fluctuation and is written as $T_{\Delta}=\hbar /\left(\mathrm{g}_{\mathrm{e}} \mu_{\mathrm{B}} \Delta\right)$ [7] where $\Delta^{2}=(2 / 3)\left\langle B_{\mathrm{f}}^{2}\right\rangle, T_{\mathrm{s}}^{\text {int }}=1 /\left(1 / \tau_{\mathrm{r}}+1 / \tau_{\mathrm{s}}^{\text {int }}\right)$ is the "intrinsic" electron spin lifetime without the electron spin-flip process, and $\gamma^{\prime}=N Q \gamma$ is the upper limit of electron spin-flip relaxation rate. Note that $T_{\mathrm{s}}$ introduced with $B_{1 / 2}$ in Eq. (6) depends on $\omega_{\mathrm{e}}$ through the electron spin-flip relaxation term.

As clearly shown, both Eqs. (7) and (8) have a Lorentzianshaped dip. The dip described by Eq. (7) originates from the effect of nuclear spin fluctuation and is characterized by $T_{\Delta}$ as well as $T_{\mathrm{s}}$. From previous works [23,27-31], the order of $T_{\Delta}$ seems to be 0.1-1 ns in SA QDs, and this value is considered to be same order as $T_{\mathrm{S}}$ with the assumption that $T_{\mathrm{s}}$ is limited by $\tau_{\mathrm{r}}$ of $\sim 1 \mathrm{~ns}$ which is shorter than $\tau_{\mathrm{s}}^{\mathrm{int}}$ [32]. Consequently, the Lorentzian dip in Eq. (7) reflects a time constant of $\lesssim 1 \mathrm{~ns}$.

On the other hand, the dip described by Eq. (8) comes from the electron spin-flip relaxation. Thus it synchronizes with $1 / T_{\mathrm{NF}}$, and is characterized by $\tau_{\mathrm{c}}$ which is often found on the order of $10 \mathrm{ps}$ in previous works. Accordingly, as compared in Fig. 4(b), the dip of Eq. (8) changes quite gradually compared to that of Eq. (7). Therefore, $\left\langle S_{z}\right\rangle$ exhibits double dip structure composed of a shallow-broad dent and a deep-spiky dip as shown in Fig. 4(c).

A blue curve in Fig. 4(d) shows the NSP formation term which has a gradual buildup originating from $1 / T_{\mathrm{NF}}$ and a sharp dip centered at $\omega_{\mathrm{e}}$. Appearance of the sharp dip, which comes from the nuclear spin fluctuation, is a striking difference from the conventional model and, as will be discussed, explains the Mid. branch. On the other hand, the electron spin-flip relaxation does not make a stark change because it follows $1 / T_{\mathrm{NF}}$, although it is necessary for reproducing the details of the experimental results.

Figures 4(d) and 4(e) give a schematic explanation for the steady-state solutions of Eq. (1), where the solutions are interpreted as the intersections of the NSP formation term (blue) and the decay term (orange). Here, Fig. 4(e) is a closeup view of the shaded region of (d). In these figures, the change in $f_{\mathrm{e}}$ affects only on the inclination of the decay term of $\left\langle I_{z}\right\rangle$; a larger $f_{\mathrm{e}}$ leads to a smaller inclination in this plot.

Starting from the lower $f_{\mathrm{e}}$ where the system takes a unique solution, the intersection follows the formation curve with increasing $f_{\mathrm{e}}$ until point $\mathrm{A}$, and it jumps to point $\mathrm{B}$. Subsequently, the state moves to point $\mathrm{C}$ along the dip structure and finally jumps to point $\mathrm{D}$ (i.e., $\mathrm{A} \rightarrow \mathrm{B} \rightarrow \mathrm{C} \rightarrow \mathrm{D}$ ). Returning from the higher $f_{\mathrm{e}}$, on the other hand, the system maintains a unique intersection till point $\mathrm{E}$, and the state-tracking route $\mathrm{E} \rightarrow \mathrm{F} \rightarrow \mathrm{G} \rightarrow \mathrm{H}$ is obtained as shown in the figure.

The trajectories of these intersections and accompanying $\left\langle S_{z}\right\rangle$ are plotted as a function of $f_{\mathrm{e}}$ in Fig. 4(f). The labeled points A-H correspond to those in Figs. 4(d) and 4(e). The loops $\mathrm{ABGH}$ and $\mathrm{CDEF}$ can be regarded as loops I and II in the observed results [Fig. 2(b)], respectively. From these considerations, we found that the plateau of the Mid. branch [B-C in the bottom panel of Fig. 4(f)] came from the narrow dip centered at $\omega_{\mathrm{e}}=0$. Since a steep decay of $\left\langle S_{z}\right\rangle$ occurs at this point as shown in the top panel, it is difficult for the NSP to grow and overcome the compensation point even under the increasing $f_{\mathrm{e}}$. As a result, the saturation of $B_{\mathrm{n}, z}^{\text {stat }}$ occurs, leading the appearance of the Mid. branch.

\section{Effects of electron spin relaxations and system parameters}

Figure 5 summarizes the impacts of each electron spin relaxation mechanism on $B_{\mathrm{n}, z}^{\text {stat }}$ and $\left\langle S_{z}\right\rangle$. Figure 5(a) shows the simplest case where $\left\langle S_{z}\right\rangle$ is treated as an input constant. Although the bistability of $B_{\mathrm{n}, z}^{\text {stat }}$ is well reproduced as reported in previous works [10-14,24], this simple condition is inadequate to explain the three stable branches of NSP as well as, of course, the synchronized change in $\left\langle S_{z}\right\rangle$. The incorporation of the electron spin-flip relaxation in Eq. (4) induces the dynamic change of $\left\langle S_{z}\right\rangle$ as shown in Fig. 5(b) where $B_{\mathrm{f}}$ is set to zero. The change in $\left\langle S_{z}\right\rangle$ synchronized with $B_{\mathrm{n}, z}^{\text {stat }}$ is obtained: the lower $\left\langle S_{z}\right\rangle$ in the Hi. branch compared with that in the Lo. branch and the gradual restoration in the Hi. branch due to the overcompensation of $B_{z}$, that is, $B_{\mathrm{n}, z}^{\text {stat }} /\left(-B_{z}\right)>1$. However, the appearance of the Mid. branch cannot be confirmed in this panel too.

The calculation in Fig. 5(c) highlights the significance of the nuclear spin fluctuation for the appearance of the Mid. branch, where the electron spin-flip relaxation is excluded. As clearly shown, the Mid. branch with a long plateau appears around the point $B_{\mathrm{n}, z}^{\text {stat }} /\left(-B_{z}\right)=1$, and a considerable reduction of $\left\langle S_{z}\right\rangle$ in the Mid. branch occurs. Furthermore, the relation $\left|B_{\mathrm{n}, z}^{\text {stat }}\right|<\left|B_{z}\right|$ in the Mid. branch is unambiguously confirmed; the stable state of the Mid. branch depicted as a solid curve lies below the dotted horizontal line, accounting for the position $B_{\mathrm{n}, z}^{\text {stat }} /\left(-B_{z}\right)=1$. These features were consistent with the experimental results shown in Fig. 2(b).

Note that the difference between $\left\langle S_{z}\right\rangle$ in the Hi. and Lo. branches is quite smaller than the observed change in $\left|\rho_{\mathrm{c}}\right|$ [Fig. 2(b)]. To resolve this discrepancy, it is required to introduce the electron spin-flip relaxation. As shown in Fig. 5(d), the incorporation of both the electron spin-flip relaxation and the influence of nonzero nuclear spin fluctuation into the model is essential for the better agreement between the experimental and theoretical results.

Next, we examine the impact of system parameters such as $B_{\mathrm{f}}$ and $\tau_{\mathrm{c}}$ on the general features of $B_{\mathrm{n}, z}^{\text {stat }}$ curve. Figure 6(a) shows the calculated $B_{\mathrm{n}, z}^{\text {stat }}$ with various $B_{\mathrm{f}}$ at a fixed $\tau_{\mathrm{c}}$. As mentioned above, the curve with $B_{\mathrm{f}}=0$ does not have the Mid. branch and thus exhibits a well-known bistable behavior. On the other hand, a nonzero $B_{\mathrm{f}}$ yields the Mid. branch of NSP; the plateau grows with increasing $B_{\mathrm{f}}$ while the overall shape is almost unchanged. Therefore, $B_{\mathrm{f}}$ affects dominantly on the local shape around the point $B_{\mathrm{n}, z}^{\text {stat }} /\left(-B_{z}\right)=1$.

Figure 6(b) indicates the impact of $\tau_{\mathrm{c}}$ at a fixed $B_{\mathrm{f}}$. We found that the width of the hysteresis loop increases with prolonging $\tau_{\mathrm{c}}$ and it manifests as a transformation from tristable to bistable behavior. Namely, the presence of the Mid. branch becomes less obvious as $\tau_{\mathrm{c}}$ increases and the plateau is buried in the large hysteresis loop in the long- $\tau_{\mathrm{c}}$ limit, which makes the experimental confirmation of three stable branches difficult. These calculations are consistent with the experimental results. That is, $B_{\mathrm{n}, z}$ in QD3 with a large hysteresis loop [Fig. 3(b)] indicated just a conventional bistable response [Fig. 3(a)], while $B_{\mathrm{n}, z}$ in QD2 with a smaller hysteresis [Fig. 3(c)] easily exhibited a distinct Mid. branch as shown in Fig. 2(b).

Looking carefully, Fig. 6(b) indicates the possibility of tristability where three stable branches exist simultaneously. 

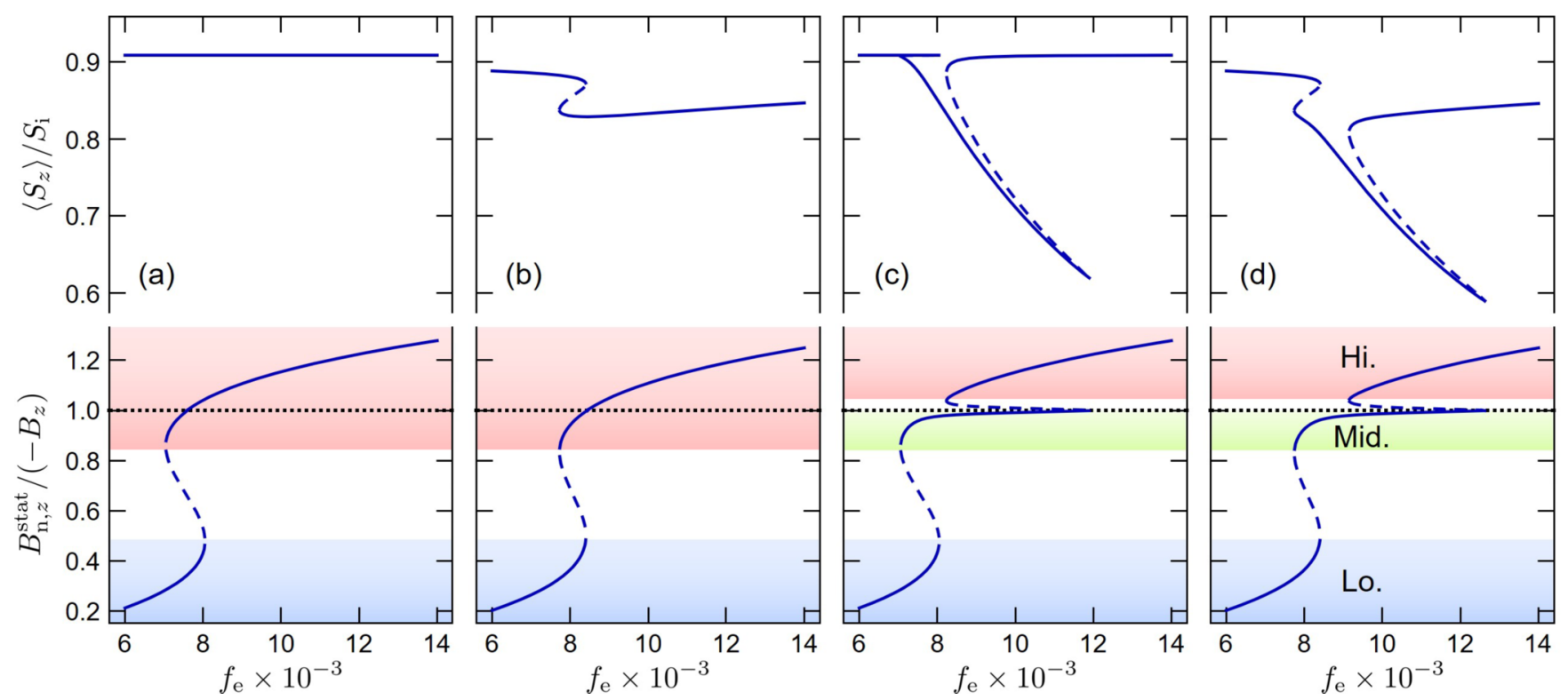

FIG. 5. The impacts of each electron spin relaxation term on the normalized $\left\langle S_{z}\right\rangle$ (top) and $B_{\mathrm{n}, z}^{\text {stat }}$ (bottom). These parameters were used in the calculations: $\tilde{A}_{z}=50 \mu \mathrm{eV}, N=3 \times 10^{4},\left\langle S_{\mathrm{eq}}\right\rangle=0, \tau_{\mathrm{c}}=17 \mathrm{ps}, T_{\mathrm{ND}}=80 \mathrm{~ms}, S_{\mathrm{i}}=-0.5, \mathrm{~g}_{\mathrm{e}}=+0.3, \tau_{\mathrm{r}}=1 \mathrm{~ns}, \tau_{\mathrm{s}}^{\text {int }}=10 \mathrm{~ns}$, and $B_{z}=$ $+5.0 \mathrm{~T}$. The dotted horizontal lines indicate the position $B_{\mathrm{n}, z}^{\text {stat }} /\left(-B_{z}\right)=1$ and the solid (dashed) blue curves represent the stable (unstable) states. (a) The simplest case where $\left\langle S_{z}\right\rangle$ is treated as a constant. (b) Impacts of the electron spin-flip relaxation with $B_{\mathrm{f}}=0$. (c) Impacts of the nuclear spin fluctuation with $B_{\mathrm{f}}=40 \mathrm{mT}$ where the electron spin-flip relaxation term is excluded. (d) Incorporation of both the electron spin-flip relaxation and nuclear spin fluctuation.

As shown in the figure, the overall shape of the calculated $B_{\mathrm{n}, z}^{\text {stat }}$ is quite sensitive to the change in $\tau_{\mathrm{c}}$, and thus, a proper choice of $\tau_{\mathrm{c}}$ is necessary to demonstrate a tristable response in
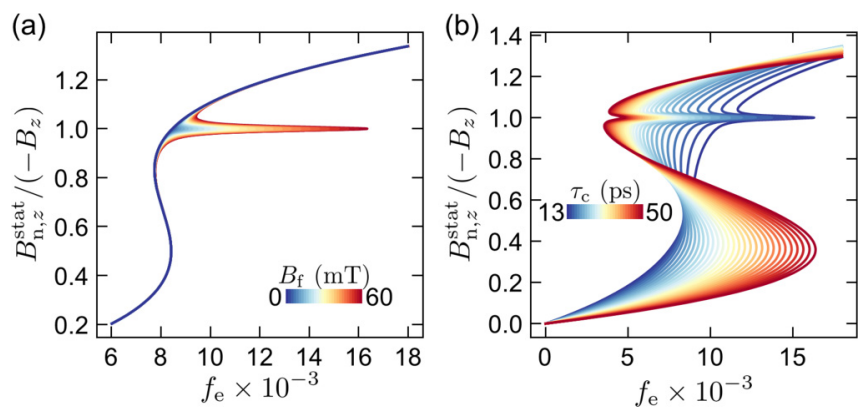

(c)

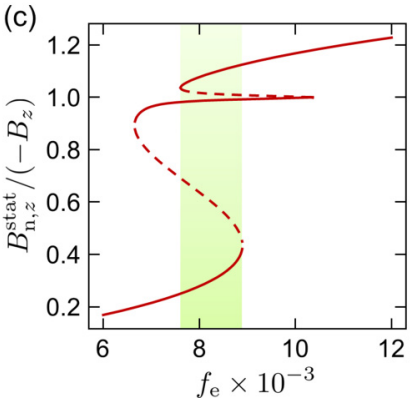

FIG. 6. The impacts of system parameters on $f_{\mathrm{e}}$ dependence of NSP. The input parameters except for $B_{\mathrm{f}}$ and $\tau_{\mathrm{c}}$ are common among the panels and are same with those in Fig. 5. Sets of calculations (a) with various $B_{\mathrm{f}}$ at a fixed $\tau_{\mathrm{c}}(=17 \mathrm{ps})$, (b) with various $\tau_{\mathrm{c}}$ at a fixed $B_{\mathrm{f}}(=40 \mathrm{mT})$. (c) Tristability of calculated NSP with a moderate set of parameters: $\tau_{\mathrm{c}}=21 \mathrm{ps}$ and $B_{\mathrm{f}}=40 \mathrm{mT}$. All stable branches appear in the highlighted region. the calculation. Figure 6(c) presents an example of tristability with a moderate set of parameters such as $\tau_{\mathrm{c}}=21 \mathrm{ps}$ and $B_{\mathrm{f}}=40 \mathrm{mT}$. In the highlighted region, all stable branches (Lo., Mid., and Hi.) coexist. Although such a tristability has not been found experimentally thus far, it can be realized if the QD system satisfies specific conditions. In particular, the control of $\tau_{\mathrm{c}}$, which has been demonstrated by changing the sample temperature [33] and the bias voltage in a charge tunable device [34], may reveal the dynamic transformation between bistable and tristable responses of NSP.

The existence of three stable branches is considered as a general property of NSP in QDs. This is because the model discussed in this paper does not include any mechanisms and parameters which refer to the specific materials or lattice structures. However, there might be some preferable systems to observe the three stable branches. For example, the magnitude of nuclear spin fluctuation is expected to depend on the QD material [7] and the reported values in InAlAs QDs [27] and InAs QDs [28,29] are larger than those in InP QDs [30] and InGaAs QDs [31]. In regard to this point, the former materials might be preferred to find the Mid. branch because the large $B_{\mathrm{f}}$ yields a long plateau as shown in Fig. 6(a).

Also, the difficulty to find the three stable branches might depend on whether the splitting between $\sigma^{+}$and $\sigma^{-}$PLs expands or shrinks when the generated nuclear field opposite to the external field changes the electron Zeeman splitting. This is determined by the relative magnitudes and signs of electron and hole $g$ factors. For instance, in InGaAs QDs, which exhibit the shrinking of PL splitting [11], it might be harder to find the third branch rather than in InAlAs QDs, which show the expanding of PL splitting [Fig. 2]. However, the third branch should be found by monitoring the change in $\rho_{\mathrm{c}}$ as shown in Fig. 3(e). 


\section{CONCLUSION}

We found the third stable branch of NSP in single InAlAs SA QDs. That implies that NSP indicates three stable branches, although the number of branches has been believed to be two at the maximum thus far. The phenomenon was tested by changing the excitation power, as well as light polarization, under a longitudinal magnetic field. The phenomenological model based on the dynamic formation of NSP was developed, including the effect of nuclear spin fluctuation, which successfully explained the three stable branches observed. Since the developed model does not relate with specific materials or lattice structures, the observed phenomenon is considered to be general in the systems where confined electron and nuclear spins coupled via HFI. Furthermore, the model predicts the tristability of NSP, which may lead to new strategies to prepare complicated QD systems, such as those involving chaotic behavior by using high degrees of freedom.

\section{ACKNOWLEDGMENT}

This work is supported by JSPS KAKENHI (Grants No. JP26800162 and No. JP17K19046).

\section{APPENDIX : DERIVATION OF EQ. (6)}

In the steady state, Eq. (3) gives

$$
S_{z}=S_{0} \frac{B_{1 / 2}^{2}+\left(B_{z}+B_{\mathrm{n}, z}^{\mathrm{stat}}+B_{\mathrm{f}, z}\right)^{2}}{B_{1 / 2}^{2}+B_{\mathrm{f}, x}^{2}+B_{\mathrm{f}, y}^{2}+\left(B_{z}+B_{\mathrm{n}, z}^{\mathrm{stat}}+B_{\mathrm{f}, z}\right)^{2}} .
$$

Here, only the $z$ component of the static nuclear field is considered because both the injected electron spin and the applied magnetic field are in the $z$ direction, and the collinear HFI induces the static NSP, hence $\boldsymbol{B}_{\mathrm{n}}^{\text {stat }}$ along $z$ axis. In addition, we have to consider that the magnitude and direction of $\boldsymbol{B}_{\mathrm{f}}$ change in each lifetime of single electron. In other words, the single electron spins existing in the QD at different times feel the different fields, and thus the averaged value of electron spin $\langle\boldsymbol{S}\rangle$ is different from the steady-state value for a single electron.

We assume a Gaussian distribution $W_{\Delta}$ of $\boldsymbol{B}_{\mathrm{f}}$ with variance of $\Delta^{2} / 2$ as follows [7]:

$$
W_{\Delta}\left(\boldsymbol{B}_{\mathrm{f}}\right)=\frac{1}{\pi^{3 / 2} \Delta^{3}} \exp \left[-\left(\frac{B_{\mathrm{f}}}{\Delta}\right)^{2}\right] .
$$

This distribution corresponds to a spatially isotropic fluctuation and $W_{\Delta}$ does not depend on any directional parameters such as azimuth and polar angles. The expectation value of $\left\langle B_{\mathrm{f}}^{2}\right\rangle$, which is used explicitly in our model, is calculated as $(3 / 2) \Delta^{2}$ with this distribution. By weighting Eq. (A1) using $W_{\Delta}$, we can obtain the averaged steady state $\left\langle S_{z}\right\rangle$ as

$$
\left\langle S_{z}\right\rangle=\int d \boldsymbol{B}_{\mathrm{f}} W_{\Delta}\left(\boldsymbol{B}_{\mathrm{f}}\right) S_{z}
$$

In this paper, by following Kuznetsova et al. [35], we use a simple approximation to avoid the complicated integral in Eq. (A3). In the approximation, we simply substitute the relations $\left\langle B_{\mathrm{f}}^{2}\right\rangle=3\left\langle B_{\mathrm{f}, \xi}^{2}\right\rangle$ and $\left\langle B_{\mathrm{f}, \xi}\right\rangle=0(\xi=x, y, z)$, and obtain Eq. (6).
[1] D. Gammon, Al. L. Efros, T. A. Kennedy, M. Rosen, D. S. Katzer, D. Park, S. W. Brown, V. L. Korenev, and I. A. Merkulov, Phys. Rev. Lett. 86, 5176 (2001).

[2] T. Yokoi, S. Adachi, H. Sasakura, S. Muto, H. Z. Song, T. Usuki, and S. Hirose, Phys. Rev. B 71, 041307(R) (2005).

[3] B. Eble, O. Krebs, A. Lemaître, K. Kowalik, A. Kudelski, P. Voisin, B. Urbaszek, X. Marie, and T. Amand, Phys. Rev. B 74, 081306(R) (2006).

[4] J. M. Taylor, C. M. Marcus, and M. D. Lukin, Phys. Rev. Lett. 90, 206803 (2003).

[5] D. A. Gangloff, G. Éthier-Majcher, C. Lang, E. V. Denning, J. H. Bodey, D. M. Jackson, E. Clarke, M. Hugues, C. Le Gall, and M. Atatüre, Science 364, 62 (2019).

[6] E. V. Denning, D. A. Gangloff, M. Atatüre, J. Mørk, and C. Le Gall, Phys. Rev. Lett. 123, 140502 (2019).

[7] I. A. Merkulov, Al. L. Efros, and M. Rosen, Phys. Rev. B 65, 205309 (2002).

[8] T. Botzem, R. P. G. McNeil, J.-M. Mol, D. Schuh, D. Bougeard, and H. Bluhm, Nat. Commun. 7, 11170 (2016).

[9] R. Stockill, C. Le Gall, C. Matthiesen, L. Huthmacher, E. Clarke, M. Hugues, and M. Atatüre, Nat. Commun. 7, 12745 (2016).
[10] P.-F. Braun, B. Urbaszek, T. Amand, X. Marie, O. Krebs, B. Eble, A. Lemaître, and P. Voisin, Phys. Rev. B 74, 245306 (2006).

[11] A. I. Tartakovskii, T. Wright, A. Russell, V. I. Falko, A. B. Van'kov, J. Skiba-Szymanska, I. Drouzas, R. S. Kolodka, M. S. Skolnick, P. W. Fry, A. Tahraoui, H.-Y. Liu, and M. Hopkinson, Phys. Rev. Lett. 98, 026806 (2007).

[12] P. Maletinsky, C. W. Lai, A. Badolato, and A. Imamoglu, Phys. Rev. B 75, 035409 (2007).

[13] R. Kaji, S. Adachi, H. Sasakura, and S. Muto, Phys. Rev. B 77, 115345 (2008).

[14] T. Belhadj, T. Kuroda, C.-M. Simon, T. Amand, T. Mano, K. Sakoda, N. Koguchi, X. Marie, and B. Urbaszek, Phys. Rev. B 78, 205325 (2008).

[15] C. Latta, A. Högele, Y. Zhao, A. N. Vamivakas, P. Maletinsky, M. Kroner, J. Dreiser, I. Carusotto, A. Badolato, D. Schuh, W. Wegscheider, M. Atature, and A. Imamoglu, Nat. Phys. 5, 758 (2009).

[16] A. Högele, M. Kroner, C. Latta, M. Claassen, I. Carusotto, C. Bulutay, and A. Imamoglu, Phys. Rev. Lett. 108, 197403 (2012). 
[17] O. Krebs, P. Maletinsky, T. Amand, B. Urbaszek, A. Lemaître, P. Voisin, X. Marie, and A. Imamoglu, Phys. Rev. Lett. 104, 056603 (2010).

[18] S. Yamamoto, R. Matsusaki, R. Kaji, and S. Adachi, Phys. Rev. B 97, 075309 (2018).

[19] H. Kumano, S. Kimura, M. Endo, H. Sasakura, S. Adachi, S. Muto, and I. Suemune, J. Nanoelectron. Optoelectron. 1, 39 (2006).

[20] Although the circularly polarized light can be prepared only by LP and QWP in principle, the additional HWP was used to cancel out the undesired phase distortion caused by optical elements such as mirror, half mirror, and/or BS in the excitation beam path.

[21] C. Testelin, F. Bernardot, B. Eble, and M. Chamarro, Phys. Rev. B 79, 195440 (2009).

[22] E. A. Chekhovich, A. Ulhaq, E. Zallo, F. Ding, O. G. Schmidt, and M. S. Skolnick, Nat. Mater. 16, 982 (2017).

[23] R. Kaji, R. Matsusaki, S. Yamamoto, and S. Adachi, Jpn. J. Appl. Phys. 58, SBBH05 (2019).

[24] B. Urbaszek, X. Marie, T. Amand, O. Krebs, P. Voisin, P. Maletinsky, A. Högele, and A. Imamoglu, Rev. Mod. Phys. 85, 79 (2013).

[25] A. Abragam, Principles of Nuclear Magnetism (Oxford University Press, Oxford, 1961), Chap. 8.

[26] O. Krebs, B. Eble, A. Lemaître, P. Voisin, B. Urbaszek, T. Amand, and X. Marie, C. R. Physique 9, 874 (2008).
[27] R. Kaji, S. Adachi, H. Sasakura, and S. Muto, Phys. Rev. B 85, 155315 (2012).

[28] P.-F. Braun, X. Marie, L. Lombez, B. Urbaszek, T. Amand, P. Renucci, V. K. Kalevich, K. V. Kavokin, O. Krebs, P. Voisin, and Y. Masumoto, Phys. Rev. Lett. 94, 116601 (2005).

[29] O. Krebs, B. Eble, A. Lemaître, B. Urbaszek, K. Kowalik, A. Kudelski, X. Marie, T. Amand, and P. Voisin, Phys. Stat. Solidi. A 204, 202 (2007).

[30] B. Pal, S. Y. Verbin, I. V. Ignatiev, M. Ikezawa, and Y. Masumoto, Phys. Rev. B 75, 125322 (2007).

[31] A. Bechtold, D. Ranuch, F. Li, T. Simmet, P.-L. Ardelt, A. Regler, K. Muller, N. A. Sinitsyn, and J. J. Finley, Nat. Phys. 11, 1005 (2015).

[32] T. Watanuki, S. Adachi, H. Sasakura, and S. Muto, Appl. Phys. Lett. 86, 063114 (2005).

[33] B. Urbaszek, P.-F. Braun, T. Amand, O. Krebs, T. Belhadj, A. Lemaître, and P. Voisin, and X. Marie, Phys. Rev. B 76, 201301(R) (2007).

[34] J. Nilsson, L. Bouet, A. J. Bennett, T. Amand, R. M. Stevenson, I. Farrer, D. A. Ritchie, S. Kunz, X. Marie, A. J. Shields, and B. Urbaszek, Phys. Rev. B 88, 085306 (2013).

[35] M. S. Kuznetsova, K. Flisinski, I. Ya. Gerlovin, I. V. Ignatiev, K. V. Kavokin, S. Yu. Verbin, D. R. Yakovlev, D. Reuter, A. D. Wieck, and M. Bayer, Phys. Rev. B 87, 235320 (2013). 